TOKYO J. MATH.

VOL. 28, No. 2, 2005

\title{
On Some Tubes over $J$-holomorphic Curves in $S^{6}$
}

\author{
Dedicated to Professor Koichi Ogiue on his 60th birthday
}

Hideya HASHIMOTO and Katsuya MASHIMO

Meijo University and Tokyo University of Agriculture and Technology

(Communicated by Y. Ohnita)

\section{Introduction}

Let $(M, g)$ be a Riemannian manifold. We denote by $G^{p}\left(T_{m} M\right)$ the Grassmann manifold of all oriented $p$-dimensional linear subspaces of the tangent space $T_{m} M$ of $M$ at $m \in M$ and by $G^{p}(T M)$ the Grassmann bundle $\bigcup_{m \in M} G^{p}\left(T_{m} M\right)$. Let $V$ be a subbundle of $G^{p}(T M)$. A $p$-dimensional submanifold $N$ of $M$ is called a $V$-submanifold if $T_{m} N \in V$ holds for any $m \in N$. If a Lie group $G$ acts on $M$, the action is naturally extended to the action of $G$ on $G^{p}(T M)$. It seems to be an interesting problem to study $V$-submanifold for an orbit $V$ of an action of $G$ on $G^{p}(T M)$.

Let $J$ be the standard almost complex structure of the 6-dimensional sphere $S^{6}$ and $\langle$,$\rangle the standard Riemannian metric. It is well-known that the group of automorphisms of$ $\left(S^{6}, J,\langle\rangle,\right)$ is isomorphic to the compact exceptional simple Lie group $G_{2}$. The complex volume form $\omega$ of the tangent space $T_{m} S^{6}$ at $m \in S^{6}$ is extended to a $G_{2}$-invariant (complex) 3 -form on $S^{6}$. For a complex number $\kappa(|\kappa| \leq 1)$, we put

$$
V_{\kappa}=\left\{\xi \in G^{3}\left(T S^{6}\right): \omega(\xi)=\kappa\right\} .
$$

A 2-dimensional submanifold $\varphi: M^{2} \rightarrow S^{6}$ is said to be a $J$-holomorphic curve if $J\left(d \varphi\left(T_{m} M\right)\right)=d \varphi\left(T_{m} M\right)$ holds for all $m \in M$. Bryant [1] showed that for any Riemann surface $M$ there exists a superminimal $J$-holomorphic curve $\varphi: M \rightarrow S^{6}$ which has no geodesic point. In this note we study whether a tube over a $J$-holomorphic curve (in the direction of first or second) normal space is a $V_{\kappa}$-submanifold or not. In the case of tubes in the direction of second normal space, we shall prove the following

THEOREM 1. Let $\varphi: M^{2} \rightarrow S^{6}$ be a J-holomorphic curve without geodesic point. If a tube $\tilde{\varphi}_{2, \gamma}$ over $\varphi$ of radius $\gamma$ is a $V_{\kappa}$-submanifold, then one of the following holds

(i) $\gamma=\pi / 2$ and $\kappa=1$,

Received June 22, 2004 
(ii) $\varphi$ is a superminimal $J$-holomorphc curve and

$$
\kappa=\cos \gamma \frac{9 \cos ^{2} \gamma-8}{4-3 \cos ^{2} \gamma}
$$

A submanifold $N$ of $S^{6}$ is said to be a totally real submanifold if $J\left(T_{m} N\right) \perp T_{m} N$ holds for any $m \in N$. A tube over a $J$-holomorphic curve $\varphi: M^{2} \rightarrow S^{6}$ is a totally real submanifold if $\varphi$ is superminimal and the radius is equal to $\arccos (\sqrt{5} / 3)$ (Ejiri [3]) and if the radius is equal to $\pi / 2$ (Dillen and Vranken [2]).

For a 3-dimensional subspace $\xi$ of $T_{m} S^{6}$ the condition $J(\xi) \perp \xi$ is equivalent to $|\omega(\xi)|=1$. The second author showed that $\omega\left(T_{m} N\right)= \pm 1(m \in N)$ holds for a 3dimensional totally real submanifolds [5], namely a totally real submanifold is nothing but a $V_{ \pm 1}$-submanifold. Recently the second author [7] proved that if a compact $V_{\kappa}$ submanifold exists then $\kappa$ is a real number.

Ejiri [3] also showed that there exists a tube $\tilde{\varphi}_{1, \gamma}$ over a $J$-holomorphic curve $\varphi: S_{1 / 6}^{2} \rightarrow$ $S^{6}$ which is a totally real submanifold. But the value, he obtained as the radius of the tube, is incorrect. In this paper, we also study tubes in the direction of the first normal bundle over $J$-holomorphic curves.

The authors would like to thank Professor Hiroyuki Tasaki for his comment on the improvement of the proof of Proposition 3.

\section{Cayley algebra}

Take an orthonormal basis $\left\{E_{0}=1, E_{1}, \cdots, E_{7}\right\}$ of the Cayley algebra $\mathfrak{C}$ such that

$$
\begin{array}{llll}
E_{1} E_{2}=E_{3}, & E_{1} E_{4}=E_{5}, & E_{1} E_{7}=E_{6}, & E_{2} E_{5}=E_{7}, \\
E_{2} E_{4}=E_{6}, & E_{3} E_{4}=E_{7}, & E_{3} E_{6}=E_{5} .
\end{array}
$$

We put

$$
\mathfrak{C}_{0}=\{u \in \mathfrak{C}: u+\bar{u}=0\}
$$

where "-" denotes the conjugation in $\mathfrak{C}$. The unit sphere $S^{6} \subset \mathfrak{C}_{0}$ centered at the origin has an almost complex structure $J$ defined by

$$
J_{m}(X)=m \cdot X, \quad m \in S^{6}, X \in T_{m} S^{6} .
$$

We denote by $G_{2}$ the group of all automorphisms of $\mathfrak{C}$. We identify $\mathfrak{C}_{0}=\sum_{i=1}^{7} \mathbf{R} E_{i}$ with the set of all 7-dimensional column vectors in a natural manner and consider $G_{2}$ as a subgroup of $S O(7)$.

LEMMA 2. For a pair of mutually orthogonal unit vectors $a_{1}, a_{2}$ in $\mathfrak{C}_{0}$ put $a_{3}=a_{1} \cdot a_{2}$. Take a unit vector $a_{4} \in \mathfrak{C}_{0}$, which is perpendicular to $a_{1}, a_{2}$ and $a_{3}$. If we put $a_{5}=a_{1} \cdot a_{4}$, 
$a_{6}=a_{2} \cdot a_{4}$ and $a_{7}=a_{3} \cdot a_{4}$ then the matrix

$$
g=\left[a_{1}, a_{2}, a_{3}, a_{4}, a_{5}, a_{6}, a_{7}\right] \in S O(7)
$$

is an element of $G_{2}$.

We denote by $\omega_{1}, \cdots, \omega_{7}$ the orthonormal coframe dual to $E_{1}, \cdots, E_{7}$. The complex volume form

$$
\omega=\left(\omega_{2}+\sqrt{-1} \omega_{3}\right) \wedge\left(\omega_{4}+\sqrt{-1} \omega_{5}\right) \wedge\left(\omega_{7}+\sqrt{-1} \omega_{6}\right) .
$$

of the tangent space $T_{E_{1}} S^{6}$ is extended to a $G_{2}$-invariant 3 -form on $S^{6}$, which we also denote by $\omega$.

Proposition 3. Let $\xi$, $\xi^{\prime}$ be elements of $G^{3}\left(T S^{6}\right)$. There exists an element $g \in G_{2}$ such that $g(\xi)=\xi^{\prime}$ if and only if $\omega(\xi)=\omega\left(\xi^{\prime}\right)$.

PROOF. Without loss of generality, we may assume that $\xi_{1}$ and $\xi_{2}$ are subspaces of $T_{E_{1}} S^{6}$.

If we take a suitable oriented base $v_{1}, v_{2}, v_{3}$ of $\xi$, the restriction of the Kähler form $\Omega(X, Y)=\langle J(X), Y\rangle$ on $\xi$ is of the form

$$
\left[\left\langle J\left(v_{i}\right), v_{j}\right\rangle\right]=\left(\begin{array}{ccc}
0 & a & 0 \\
-a & 0 & 0 \\
0 & 0 & 0
\end{array}\right), \quad a \geq 0 .
$$

There exists an element $h \in S U(3)$ such that

$$
h\left(v_{1}\right)=E_{2}, \quad h\left(v_{2}\right)=a E_{3}+b E_{4}+c E_{5}, \quad h\left(v_{3}\right)=E_{7} .
$$

Note that $\omega(\xi)=b+\sqrt{-1} c$. We take an oriented orthonormal base $v_{1}^{\prime}, v_{2}^{\prime}, v_{3}^{\prime}$ of $\xi^{\prime}$ and $h^{\prime} \in S U(3)$ with

$$
h^{\prime}\left(v_{1}^{\prime}\right)=E_{2}, \quad h^{\prime}\left(v_{2}^{\prime}\right)=a^{\prime} E_{3}+b^{\prime} E_{4}+c^{\prime} E_{5}, \quad h^{\prime}\left(v_{3}^{\prime}\right)=E_{7} .
$$

Since the only element of $S U(3)$ which stabilizes $E_{2}$ and $E_{7}$ is the unit element, there exists an element $g \in S U(3)$ with $g(\xi)=\xi^{\prime}$ if and only if $(a, b, c)=\left(a^{\prime}, b^{\prime}, c^{\prime}\right)$.

q.e.d.

\section{2. $J$-holomorphic curve in $S^{6}$}

Let $\varphi: M^{2} \rightarrow S^{6}$ be a $J$-holomorphic curve in $S^{6}$. We denote by $\sigma$ the second fundamental form of a $J$-holomorphic curve $\varphi$. Take an orthonormal frame $e_{1}, e_{2}=J\left(e_{1}\right)$ of $T_{m} M$. The length of $\sigma\left(e_{1}, e_{1}\right)_{m}$ and $\sigma\left(e_{1}, e_{2}\right)_{m}$ are equal to each other if the point $m$ is not a geodesic point. Assume that $\varphi: M \rightarrow S^{6}$ is a $J$-holomorphic curve without geodesic point and put

$$
e_{3}=\sigma\left(e_{1}, e_{1}\right) / \lambda, \quad e_{4}=\sigma\left(e_{1}, e_{2}\right) / \lambda, \quad e_{5}=e_{1} e_{3}, \quad e_{6}=J\left(e_{5}\right)
$$


where $\lambda=\left|\sigma\left(e_{1}, e_{1}\right)\right|$. Since we have $\varphi(m) \times e_{3}=e_{4}$ and $e_{2} \times e_{3}=-e_{6}$, the matrix

$$
\left[\varphi(m), e_{1}, e_{2}, e_{3}, e_{4}, e_{5},-e_{6}\right]
$$

is an element of $G_{2}$ by Lemma 2 .

We denote by $U N_{1}$ the bundle of all unit vectors in the first normal space. For a constant $\gamma$, we define the tube $\tilde{\varphi}_{1, \gamma}$ of radius $\gamma$ in the direction of the 1-st normal bundle as follows;

$$
\tilde{\varphi}_{1, \gamma}: U N_{1} \rightarrow S^{6} ;(m, X) \mapsto(\cos \gamma) \varphi(m)+\sin \gamma X .
$$

The tube $\tilde{\varphi}_{2, \gamma}$ of radius $\gamma$ in the direction of the 2 -nd normal bundle is defined in a similar fashion.

Define a (local) lift $g: M \rightarrow G_{2}$ of $\varphi$ by

$$
g(m)=\left[e_{0}, e_{1}, e_{2}, e_{3}, e_{4}, e_{5},-e_{6}\right]
$$

where we put $e_{0}=\varphi(m)$. We denote by $D$ the covariant derivative of $\mathbf{R}^{7}$ and by $\omega_{B A}$ the connection form,

$$
\omega_{B A}(X)=\left\langle D_{X} e_{A}, e_{B}\right\rangle, \quad 0 \leq A, B \leq 6 .
$$

Put $h_{i A}^{B}=\left\langle D_{e_{i}} e_{A}, e_{B}\right\rangle=\omega_{B A}\left(e_{i}\right)$. The pull-back of the Maurer-Cartan form of $G_{2}$ by $g$ is of the form

$$
\left(\begin{array}{ccccccc}
0 & \omega_{01} & \omega_{02} & \omega_{03} & 0 & 0 & 0 \\
\omega_{10} & 0 & \omega_{12} & \omega_{13} & \omega_{14} & \omega_{15} & -\omega_{16} \\
\omega_{20} & \omega_{21} & 0 & \omega_{23} & \omega_{24} & \omega_{25} & -\omega_{26} \\
\omega_{30} & \omega_{31} & \omega_{32} & 0 & \omega_{34} & \omega_{35} & -\omega_{36} \\
0 & \omega_{41} & \omega_{42} & \omega_{43} & 0 & \omega_{45} & -\omega_{46} \\
0 & \omega_{51} & \omega_{52} & \omega_{53} & \omega_{54} & 0 & -\omega_{56} \\
0 & -\omega_{61} & -\omega_{62} & -\omega_{63} & -\omega_{64} & -\omega_{65} & 0
\end{array}\right)
$$

Since (1) is a $\mathfrak{g}_{2}$-valued 1-form, we have the following

PROPOSITION 4. The connection form $\left\{\omega_{B A}\right\}$ satisfy the following

$$
\left\{\begin{array}{l}
\omega_{41}=-\omega_{32} \\
\omega_{42}=\omega_{31} \\
\omega_{62}=\omega_{51} \\
\omega_{61}=-\omega_{52} \\
\omega_{63}=-\omega_{54}+\omega_{1} \\
\omega_{64}=-\omega_{53}-\omega_{2} \\
\omega_{65}=-\omega_{43}-\omega_{21} .
\end{array}\right.
$$


In other words,

$$
\left\{\begin{array}{l}
h_{12}^{4}=h_{21}^{4}=h_{11}^{3}=-h_{22}^{3}(=\lambda), \\
h_{12}^{3}=h_{21}^{3}=-h_{11}^{4}=h_{22}^{4}=0, \\
h_{13}^{5}=h_{14}^{6}=-h_{24}^{5}=h_{23}^{6}, \\
h_{13}^{6}+h_{14}^{5}=h_{23}^{5}-h_{24}^{6}=1, \\
h_{13}^{6}+h_{24}^{6}=h_{23}^{5}-h_{14}^{5}=0 .
\end{array}\right.
$$

holds. Especially, $\varphi$ is a minimal immersion.

The third fundamental form $\sigma_{3}$ of $\varphi$ is defined as follows

$$
\sigma_{3}(u, v, w)=\left\langle D_{u} \sigma(V, W), e_{5}\right\rangle e_{5}+\left\langle D_{u} \sigma(V, W), e_{6}\right\rangle e_{6}, \quad u, v, w \in T_{m} M,
$$

where $V, W$ are locally defined vector field on $M$ with $V_{m}=v$ and $W_{m}=w$. A 2-dimensional submanifold $M$ of $S^{6}$ is said to be a superminimal surface if and only if $\left\{\sigma_{3}(X, X, X): X \in T_{m} M,|X|=1\right\}$ is a circle in the second normal space. The condition that a surface $\varphi: M^{2} \rightarrow S^{6}$ is a superminimal surface is equivalent to

$$
h_{13}^{5}+h_{23}^{6}=h_{23}^{5}-h_{13}^{6}=0 .
$$

Thus we have the following

COROLlary 5. A J-holomorphic curve $\varphi: M \rightarrow S^{6}$ without geodesic point is a superminimal surface if and only if

$$
\begin{aligned}
& h_{13}^{5}=h_{14}^{6}=h_{23}^{6}=-h_{24}^{5}=0, \\
& h_{13}^{6}=h_{14}^{5}=h_{23}^{5}=-h_{24}^{6}=1 / 2 .
\end{aligned}
$$

REMARK 6. If we put $\hat{e}_{1}=e_{2}$ and $\hat{e}_{2}=e_{1}$ and put $\hat{e}_{3}=\sigma\left(\hat{e}_{1}, \hat{e}_{1}\right) / \lambda, \hat{h}_{13}^{5}=$ $\left\langle D_{\hat{e}_{1}} \hat{e}_{3}, \hat{e}_{5}\right\rangle$ etc., then we have

$$
\hat{h}_{13}^{5}=h_{13}^{5}, \quad \hat{h}_{14}^{5}=1-h_{14}^{5} .
$$

From proposition 4 , if we put $h_{13}^{5}=v$ and $h_{14}^{5}-1 / 2=\mu$, we have

$$
\begin{aligned}
& \omega_{53}=v \omega_{1}+(\mu+1 / 2) \omega_{2} \\
& \omega_{63}=-(\mu-1 / 2) \omega_{1}+v \omega_{2} \\
& \omega_{54}=(\mu+1 / 2) \omega_{1}-v \omega_{2} \\
& \omega_{64}=v \omega_{1}+(\mu-1 / 2) \omega_{2}
\end{aligned}
$$

From the Maurer-Cartan equation of $G_{2}$, we have the following 
PROPOSITION 7 (Integrability condition).

$$
\begin{aligned}
d \omega_{1} & =\omega_{21} \wedge \omega_{2} \\
d \omega_{2} & =\omega_{12} \wedge \omega_{1} \\
d \omega_{21} & =\left(2 \lambda^{2}-1\right) \omega_{1} \wedge \omega_{2} \\
d \omega_{43} & =-\left(2 \lambda^{2}-2 \mu^{2}+2 \mu-1-2 v^{2}\right) \omega_{1} \wedge \omega_{2} \\
0 & =J^{*} d \log \lambda+2 \omega_{21}-\omega_{43} \\
0 & =J^{*} d \log \mu+2 \omega_{21}+2 \omega_{43}
\end{aligned}
$$

where $J^{*}$ is defined for 1 form by $J^{*} \alpha(X)=-\alpha(J(X)), X \in T M$.

COROLlaRY 8. ([4])

$$
\begin{aligned}
\Delta \log \lambda & =5 / 2-6 \lambda^{2}+2|\mathbf{I I I}|^{2} \\
\Delta \log |\mathbf{I I I}| & =1-4|\mathbf{I I I}|^{2}
\end{aligned}
$$

where $|\mathbf{I I I}|$ is the square of the length of the third fundamental form $|\mathbf{I I I}|^{2}=(\mu-1 / 2)^{2}+v^{2}$.

\section{Proof of theorem 1}

Let $\varphi: M \rightarrow S^{6}$ be a $J$-holomorphic curve without geodesic point. From lemma 2 an orthogonal transformation $g$ on $\mathfrak{C}_{0}$ which is defined by

$$
\begin{aligned}
& g(\varphi(x))=E_{1}, \quad g\left(e_{1}\right)=E_{2}, \quad g\left(e_{2}\right)=E_{3}, \quad g\left(e_{3}\right)=E_{4}, \\
& g\left(e_{4}\right)=E_{5}, \quad g\left(e_{5}\right)=E_{6}, \quad g\left(e_{6}\right)=-E_{7}
\end{aligned}
$$

is an element of $G_{2}$. We put $\alpha=\cos \gamma, \beta=\sin \gamma$ and

$$
A=h_{13}^{5} \cos t+h_{13}^{6} \sin t, \quad B=\left(1-h_{13}^{6}\right) \cos t+h_{13}^{5} \sin t .
$$

The tangent space of the tube

$$
\tilde{\varphi}_{2, \gamma}: U N_{2} \rightarrow S^{6}
$$

at $g^{-1}\left(\alpha E_{1}+\beta\left(\cos t E_{6}-\sin t E_{7}\right)\right)$ is spanned by

$$
\begin{aligned}
& U_{1}=g^{-1}\left({ }^{t}[0,0,0,0,0, \sin t, \cos t]\right), \\
& U_{2}=g^{-1}\left({ }^{t}[0, \alpha, 0,-\beta A,-\beta B, 0,0]\right), \\
& U_{3}=g^{-1}\left({ }^{t}[0,0, \alpha,-\beta B, \quad \beta A, 0,0]\right) .
\end{aligned}
$$


The orthogonal matrix

$$
h=\left(\begin{array}{ccccccc}
\alpha & 0 & 0 & 0 & 0 & \beta \cos t & -\beta \sin t \\
0 & 0 & 0 & 0 & 0 & \sin t & \cos t \\
-\beta & 0 & 0 & 0 & 0 & \alpha \cos t & -\alpha \sin t \\
0 & 1 & 0 & 0 & 0 & 0 & 0 \\
0 & 0 & \alpha & \beta \cos t & -\beta \sin t & 0 & 0 \\
0 & 0 & 0 & \sin t & \cos t & 0 & 0 \\
0 & 0 & -\beta & \alpha \cos t & -\alpha \sin t & 0 & 0
\end{array}\right)
$$

is an element of $G_{2}$ with $h\left(\alpha E_{1}+\beta\left(\cos t E_{6}-\sin t E_{7}\right)=E_{1}\right.$. Substiting $h\left(g\left(U_{1}\right)\right), h\left(g\left(U_{2}\right)\right)$, $h\left(g\left(U_{3}\right)\right)$ into $\left.\omega\right|_{E_{1}}$, we have

$$
\begin{aligned}
& \omega\left(U_{1}, U_{2}, U_{3}\right) \\
&= \beta\left\{\left(1 / 2+\left(h_{13}^{5}\right)^{2}-h_{13}^{6}+\left(h_{13}^{6}\right)^{2}\right) \beta^{2}-2 \alpha^{2}\right\} \\
&+\beta\left(1-3 \alpha^{2}\right)\left\{\left(1 / 2-h_{13}^{6}\right) \cos (2 t)+h_{13}^{5} \sin (2 t)\right\} \\
&+2 \sqrt{-1} \alpha \beta\left\{h_{13}^{5} \cos (2 t)+\left(h_{13}^{6}-1 / 2\right) \sin (2 t)\right\} .
\end{aligned}
$$

If we put $G=\operatorname{det}\left(\left[\left\langle U_{i}, U_{j}\right\rangle\right]\right)$, we have

$$
\omega\left(d \tilde{\varphi}_{1, \gamma}\left(T\left(U N_{2}\right)\right)=\omega\left(U_{1}, U_{2}, U_{3}\right) / \sqrt{G} .\right.
$$

The imaginary part of $\omega\left(d \tilde{\varphi}_{2, \gamma}\left(T\left(U N_{2}\right)\right)\right.$ is equal to

$$
2 \alpha \beta \frac{h_{13}^{5} \cos (2 t)+\left(h_{13}^{6}-1 / 2\right) \sin (2 t)}{\left(h_{13}^{5}\right)^{2}+\left(h_{13}^{6}\right)^{2}+h_{13}^{6}-1 / 2-\left(h_{13}^{6}-1 / 2\right) \cos (2 t)+h_{13}^{5} \sin (2 t)},
$$

which is a constant if and only if one of the following holds.

- $\alpha=\cos \gamma=0$,

- $h_{13}^{6}=1 / 2, h_{13}^{5}=0$.

If $\alpha=0$ then $\omega\left(d \tilde{\varphi}_{2, \gamma}\left(T\left(U N_{2}\right)\right)\right)=1$, namely the tube $\left.\tilde{\varphi}_{2, \pi / 2}\left(U N_{2}\right)\right)$ is a $V_{1}$ (in other word, totally real) submanifold.

If $h_{13}^{6}=1 / 2$ and $h_{13}^{5}=0$ then $\omega\left(d \tilde{\varphi}_{2, \gamma}\left(T\left(U N_{2}\right)\right)\right)$ is equal to

$$
\cos \gamma \frac{9 \cos ^{2} \gamma-8}{4-3 \cos ^{2} \gamma}
$$

q.e.d.

\section{Tubes $\varphi_{1, \gamma}$}

First we give two typical examples of $J$-holomorphic curves of $S^{6}$. 
EXAMPLE 1. The subgroup

$$
T=\left\{\exp \left(a G_{23}+b G_{45}+c G_{76}\right): a, b, c \in \mathbf{R}, a+b+c=0\right\}
$$

is a maximal torus of $G_{2}$, where $G_{i j}(1 \leq i \neq j \leq 7)$ are skew-symmetric transformations on $\mathfrak{C}_{0}$ defined by

$$
G_{i j}\left(E_{k}\right)=\delta_{j k} E_{i}-\delta_{i k} E_{j}, \quad 1 \leq k \leq 7
$$

The mapping

$$
\Phi_{0}: T \rightarrow S^{6} ;(a, b, c) \mapsto \exp \left(a G_{23}+b G_{45}+c G_{76}\right)\left(p_{0}\right)
$$

where we put $p_{0}=\left(E_{2}+E_{4}+E_{6}\right) / \sqrt{3}$, is a $J$-holomorphic curve. Take

$$
e_{1}=\sqrt{3 / 2}\left(G_{23}-G_{76}\right), e_{2}=\sqrt{1 / 2}\left(G_{23}-2 G_{45}+G_{76}\right)
$$

as a basis of the Lie algebra of $T$. We have

$$
D_{e_{1}} e_{1}=-p_{0}+\sqrt{1 / 2} e_{3}, \quad D_{e_{1}} e_{2}=\sqrt{1 / 2} e_{4}, \quad D_{e_{2}} e_{2}=-p_{0}-\sqrt{1 / 2} e_{3},
$$

where we put

$$
e_{3}=-\sqrt{1 / 6}\left(E_{2}-2 E_{4}+E_{6}\right), \quad e_{4}=-\sqrt{1 / 2}\left(E_{2}-E_{6}\right) .
$$

We extend $e_{3}, e_{4}$ to a left invariant vector fields on $T$, and denote them also by $e_{3}, e_{4}$. Put

$$
e_{5}=\sqrt{1 / 3}\left(E_{3}+E_{5}-E_{7}\right), \quad e_{6}=E_{1},
$$

and extend $e_{5}, e_{6}$ to a left invariant vector fields on $T$, and denote them also by $e_{5}, e_{6}$. We have

$$
\left\{\begin{array}{l}
h_{12}^{4}=h_{21}^{4}=h_{11}^{3}=-h_{22}^{3}=\sqrt{1 / 2}, \\
h_{12}^{3}=h_{21}^{3}=h_{22}^{4}=-h_{11}^{4}=0, \\
h_{13}^{5}=h_{14}^{6}=h_{23}^{6}=-h_{24}^{5}=0, \\
h_{14}^{5}=h_{23}^{5}=1, \quad h_{13}^{6}=h_{24}^{6}=0 .
\end{array}\right.
$$

EXAMPLE 2. There exists a 3-dimensional simple Lie subgroup $U_{4}$ of $G_{2}(\subset$ $S O\left(\mathbf{R}^{7}\right)$ ) whose action on $\mathbf{R}^{7}$ is irreducible (cf. [5]). The orbit of the subgroup $U_{4}$ through $e_{1}$ is the Veronese embedding $\Phi_{1}: S_{1 / 6}^{2} \rightarrow S^{6}$. The length of second fundamental form of $\Phi_{0}$ is $5 / 3$.

THEOREM 9. Let $\varphi: M^{2} \rightarrow S^{6}$ be a J-holomorphic curve without geodesic point. If a tube $\tilde{\varphi}_{1, \gamma}$ over $\varphi$ of radius $\gamma$ is a $V_{\kappa}$-submanifold, then one of the following holds 
(i) $\varphi$ is (locally) congruent (up to $\left.G_{2}\right)$ to the $J$-holomorphic curve $\Phi_{0}: T^{2} \rightarrow S^{5}(\subset$ $\left.S^{6}\right)$ and $\gamma=\pi / 2$. In this case $\kappa^{2}=1 / 3$.

(ii) $\varphi$ is (locally) congruent (up to $G_{2}$ ) to $\Phi_{1}: S_{1 / 6}^{2} \rightarrow S^{6}$ and $0<\gamma \leq \pi / 2$. In this case

$$
\kappa=\sin \gamma \frac{6 \cos ^{2} \gamma-2 \sin ^{2} \gamma}{\sqrt{9 \cos ^{4} \gamma-3 \cos ^{2} \gamma \sin ^{2} \gamma+4 \sin ^{4} \gamma}},
$$

(iii) $\varphi$ is a superminimal $J$-holomorphc curve $M \rightarrow S^{6}$ and $\gamma=\pi / 2$. In this case $\kappa=1$.

PROOF OF THEOREM 9. The tangent space of the tube $\tilde{\varphi}_{1, \gamma}: U N_{1} \rightarrow S^{6}$ at $g^{-1}\left(\alpha E_{1}+\right.$ $\left.\beta\left(\cos t E_{3}+\sin t E_{4}\right)\right)$ is spanned by

$$
U_{1}=g^{-1} \cdot\left(\begin{array}{c}
0 \\
0 \\
0 \\
\sin t \\
-\cos t \\
0 \\
0
\end{array}\right),
$$


We put

$$
h=\left(\begin{array}{ccccccc}
\alpha & 0 & 0 & \beta \cos t & \beta \sin t & 0 & 0 \\
0 & 0 & 0 & -\sin t & \cos t & 0 & 0 \\
\beta & 0 & 0 & -\alpha \cos t & -\alpha \sin t & 0 & 0 \\
0 & 1 & 0 & 0 & 0 & 0 & 0 \\
0 & 0 & \alpha & 0 & 0 & -\beta \cos t & -\beta \sin t \\
0 & 0 & 0 & 0 & 0 & \sin t & -\cos t \\
0 & 0 & \beta & 0 & 0 & \alpha \cos t & \alpha \sin t
\end{array}\right)
$$

and substitute $h\left(g\left(U_{1}\right)\right), h\left(g\left(U_{2}\right)\right)$ and $h\left(g\left(U_{3}\right)\right)$ into $\left.\omega\right|_{E_{1}}$. By a long (and tedious) calculation we obtain

$$
\begin{aligned}
\omega\left(U_{1}, U_{2}, U_{3}\right)= & \beta\left\{2 \alpha^{2}+\beta^{2}\left(\left(h_{13}^{5}\right)^{2}+\left(h_{14}^{5}\right)^{2}-h_{14}^{5}-\left(h_{11}^{3}\right)^{2}\right\}\right. \\
& +2 \alpha \beta^{2} h_{11}^{3} h_{13}^{5} \sin (3 t)-\alpha \beta^{2} h_{11}^{3}\left(2 h_{14}^{5}-1\right) \cos (3 t) \\
& -2 \sqrt{-1} \beta^{2} h_{11}^{3} h_{13}^{5} \cos (3 t) .
\end{aligned}
$$

If we put $G=\operatorname{det}\left(\left[\left\langle U_{i}, U_{j}\right\rangle\right]\right)$, we have

$$
\omega\left(d \tilde{\varphi}_{1, \gamma}\left(T\left(U N_{1}\right)\right)\right)=\omega\left(U_{1}, U_{2}, U_{3}\right) / \sqrt{G} .
$$

Fix a point $m$ and assume that $\omega\left(d \tilde{\varphi}_{1, \gamma}\left(T\left(U N_{1}\right)\right)\right)$ is a constant with respect to $t$. Since $G$ does not contain any term $\cos (k t)$ and $\sin (k t)$ for $k \geq 5$, the coefficient of $\sqrt{-1} \sin (6 t)$ in the expansion of $\operatorname{Im}\left(\omega\left(U_{1}, U_{2}, U_{3}\right)\right)^{2}$ is equal to 0 , namely we have

$$
2 \alpha \beta^{4}\left(h_{11}^{3}\right)^{2}\left(h_{13}^{5}\right)^{2}=0 .
$$

From the condition that the coefficient of $\sqrt{-1} \sin (6 t)$ in the expansion of $\operatorname{Im}\left(\omega\left(U_{1}, U_{2}, U_{3}\right)\right)^{2}$ is equal to 0 , we have the following two cases:

- $h_{13}^{5}=0, h_{14}^{5}=1 / 2$,

- $h_{13}^{5}=0, \alpha=0$.

CASE 1. Assume that $h_{13}^{5}=0, h_{14}^{5}=1 / 2$ hold, namely $\varphi$ is a superminimal surface. Since $\omega$ and $G$ are

$$
\begin{aligned}
& \omega\left(U_{1}, U_{2}, U_{3}\right)=\beta\left(2 \alpha^{2}-\beta^{2}\left(\lambda^{2}+1 / 4\right)\right), \\
& G=\left(\alpha^{2}+\beta^{2}\left(\lambda^{2}+1 / 4\right)\right)^{2}-(2 \lambda \alpha \beta)^{2} .
\end{aligned}
$$

the function $\kappa(m)=\omega\left(d \tilde{\varphi}_{1, \gamma}\left(T\left(U N_{1}\right)\right)\right)$ is a constant on $M$ if and only if $\lambda=h_{11}^{3}$ is a constant.

From the equation of Gauss, $M$ is a space of constant curvature. By results of Sekigawa [6] and Hashimoto [4] $\varphi$ is the Veronese surface $S_{1 / 6}^{2} \rightarrow S^{6}$ (where $\lambda=\sqrt{5 / 12}$ ). In this case, 
we have

$$
\kappa=\frac{\beta\left(6 \alpha^{2}-2 \beta^{2}\right)}{\sqrt{\left(3 \alpha^{2}+2 \beta^{2}\right)^{2}-15(\alpha \beta)^{2}}} .
$$

CASE 2. Assume that $\alpha=0$ and $h_{13}^{5}=0$. We have

$$
\kappa^{2}=\frac{\left(\lambda^{2}-\mu^{2}+1 / 4\right)^{2}}{\left(\lambda^{2}+\left(\mu^{2}+1 / 2\right)^{2}\right)\left(\lambda^{2}+\left(\mu^{2}-1 / 2\right)^{2}\right)}
$$

where we put $\lambda=h_{11}^{3}$ and $\mu=h_{14}^{5}-1 / 2$. From

$$
d \kappa^{2}=\frac{8 \lambda \mu\left(\lambda^{2}-\mu^{2}+1 / 4\right)\left\{\mu\left(\lambda^{2}+\mu^{2}-1 / 4\right) d \lambda-\lambda\left(\lambda^{2}+\mu^{2}+1 / 4\right) d \mu\right\}}{\left(\lambda^{2}+\left(\mu^{2}+1 / 2\right)^{2}\right)\left(\lambda^{2}+\left(\mu^{2}-1 / 2\right)^{2}\right)}
$$

the function $\kappa^{2}$ on $M$ is a constant if and only if one of the following holds

$$
\begin{aligned}
& 0=\mu \\
& 0=\lambda^{2}-\mu^{2}+1 / 4 \\
& 0=\mu\left(\lambda^{2}+\mu^{2}-1 / 4\right) d \lambda-\lambda\left(\lambda^{2}+\mu^{2}+1 / 4\right) d \mu .
\end{aligned}
$$

Put $M_{1}=\{m \in M: \mu(m) \neq 0\}$.

In the interior of $M \backslash M_{1}$ it falls into Case 1. So we assume that $M_{1}$ is non-empty. Since (5.2) or (5.3) holds on $M_{1}$,

$$
U=\frac{\lambda^{2}-\mu^{2}+1 / 4}{\lambda \mu}
$$

is a constant (the numerator of the differential $d U$ is equal to the left hand side of (5.3)).

From (3.5) and (3.6), we have

$$
\omega_{21}=-(1 / 6) J^{*} d \log \left|\lambda^{2} \mu\right| .
$$

Since $\omega_{21}=J^{*} d \log \rho$, we conclude that $\rho^{6} \lambda^{2} \mu$ is a constant.

Put $M_{2}=\left\{m \in M_{1}: d \mu_{m} \neq 0\right\}, M_{3}=\left\{m \in M_{2}: d \lambda_{m} \neq 0\right\}$ and assume that $M_{3}$ is not an empty set.

Since $U=\left(\lambda^{2}-\mu^{2}+1 / 4\right) / \lambda \mu$ and $V=\rho^{6} \lambda^{2} \mu$ are constants, $\lambda$ and $\mu$ are functions of $\rho$ on $M_{3}$. Namely there exist functions $\hat{\lambda}, \hat{\mu}$ of variable $\rho$ such that

$$
\lambda(x)=\hat{\lambda}(\rho(x)), \quad \mu(x)=\hat{\mu}(\rho(x)), \quad m \in M_{3} .
$$

From $\rho^{6} \lambda^{2} \mu=$ const., we have

$$
2 \rho^{2} \mu d \lambda+\rho \lambda d \mu=-6 \lambda \mu d \rho .
$$

From (5.3) and (6), we have

$$
\hat{\lambda}^{\prime}=-6 \hat{\lambda}\left(\hat{\lambda}^{2}+\hat{\mu}^{2}+1 / 4\right) / \rho\left(3 \hat{\lambda}^{2}+3 \hat{\mu}^{2}+1 / 4\right)
$$




$$
\hat{\mu}^{\prime}=-6 \hat{\mu}\left(\hat{\lambda}^{2}+\hat{\mu}^{2}-1 / 4\right) / \rho\left(3 \hat{\lambda}^{2}+3 \hat{\mu}^{2}+1 / 4\right)
$$

If $f$ is a function of $\rho$, i.e., there exists a function $F$ of 1 -variable such that $f(x)=$ $F(\rho(x)), m \in M$, we have

$$
\left(F F^{\prime \prime}-F^{\prime 2}+(1 / \rho) F F^{\prime}\right)\|\operatorname{grad} \rho\|^{2}=F^{2} \Delta \log f-F F^{\prime} \rho\left(2 \lambda^{2}-1\right) .
$$

Apply the above to $\lambda$ and $\mu$, we have

$$
\begin{aligned}
\left(\hat{\lambda} \hat{\lambda}^{\prime \prime}\right. & \left.-\hat{\lambda}^{\prime 2}+(1 / \rho) \hat{\lambda} \hat{\lambda}^{\prime}\right)\|\operatorname{grad} \rho\|^{2} \\
& =\hat{\lambda}^{2}\left(5 / 2-6 \hat{\lambda}^{2}+2 \hat{\mu}^{2}\right)-\hat{\lambda} \hat{\lambda}^{\prime} \rho\left(2 \hat{\lambda}^{2}-1\right) \\
\left(\hat{\mu} \hat{\mu}^{\prime \prime}-\right. & \left.\hat{\mu}^{\prime 2}+(1 / \rho) \hat{\mu} \hat{\mu}^{\prime}\right)\|\operatorname{grad} \rho\|^{2} \\
& =\hat{\mu}^{2}\left(1-4 \hat{\mu}^{2}\right)-\hat{\mu} \hat{\mu}^{\prime} \rho\left(2 \hat{\lambda}^{2}-1\right)
\end{aligned}
$$

From (7.1), (7.2) we have

$$
\begin{aligned}
\hat{\lambda} \hat{\lambda}^{\prime \prime}-\hat{\lambda}^{\prime 2}+(1 / \rho) \hat{\lambda} \hat{\lambda}^{\prime} & =\frac{-6 \hat{\lambda}^{2}}{\rho\left(3 \hat{\lambda}^{2}+3 \mu^{2}+1 / 4\right)^{2}}\left(\hat{\lambda} \hat{\lambda}^{\prime}+M M^{\prime}\right), \\
\hat{\mu} \hat{\mu}^{\prime \prime}-\hat{\mu}^{\prime 2}+(1 / \rho) \hat{\mu} \hat{\mu}^{\prime} & =\frac{-12 \hat{\mu}^{2}}{\rho\left(3 \hat{\lambda}^{2}+3 \mu^{2}+1 / 4\right)^{2}}\left(\hat{\lambda} \hat{\lambda}^{\prime}+\hat{\mu} \hat{\mu}^{\prime}\right) .
\end{aligned}
$$

Thus we obtain

$$
2 \hat{\mu}^{2} \times(\text { l.h.s. of }(8.1))-\hat{\lambda}^{2} \times(\text { l.h.s. of }(8.2))=0
$$

On the other hand, we have

$$
\begin{aligned}
0 & =2 \hat{\mu}^{2} \times(\text { r.h.s. of }(8.1))-\hat{\lambda}^{2} \times(\text { r.h.s. of }(8.2)) \\
& =\frac{\hat{\lambda}^{2} \hat{\mu}^{2}}{2\left(3 \hat{\lambda}^{2}+3 \hat{\mu}^{2}+1 / 4\right)}\left(48 \hat{\lambda}^{2} \hat{\mu}^{2}-12 \hat{\lambda}^{2}+48 \hat{\mu}^{4}-8 \hat{\mu}^{2}-1\right) \\
& =\frac{\hat{\lambda}^{2} \hat{\mu}^{2}}{2\left(3 \hat{\lambda}^{2}+3 \hat{\mu}^{2}+1 / 4\right)}\left(4 \hat{\mu}^{2}-1\right)\left(12 \hat{\mu}^{2}+12 \hat{\lambda}^{2}+1\right)
\end{aligned}
$$

Thus $M$ is a constant, which contradicts to our assumption $d \mu \neq 0$. Namely $M_{3}$ is an empty set and $\lambda$ is a constant on $M_{2}$. But using (5.3), we conclude that $M_{2}$ is also an empty set. Namely $\lambda$ and $\mu$ are constants on $M_{1}$. Using Corollaly 8 , we conclude that $\mu=1$ on $M_{1}$. After all if $M_{1}$ is not an empty set then $\mu=1$ on $M$ and $\lambda$ is a constant on $M$. Thus we have $K=1-2 \lambda^{2}=0$ and $\varphi$ is congruent (up to $G_{2}$ ) to $\Phi_{0}$ (cf., [4]).

q.e.d.

\section{References}

[1] BRyAnt, R. L., Submanifolds and special structures on the octonians, J. Diff. Geometry,17 (1982), $185-232$.

[2] Dillen, F. and VRancken, L., Totally real submanifolds in $S^{6}(1)$ satisfying Chen's equality, Trans. Amer. Math. Soc., 348 (1996), 1633-1646. 
[ 3 ] EJIRI, N., Equivariant minimal immersions of $S^{2}$ into $S^{2 m}$ (1), Trans. A. M. S., 297 (1986), 105-124.

[ 4 ] Hashimoto, H., J-holomorphic curves in a 6-dimensional sphere, Tokyo J. Math., 23 (2000), 137-159.

[ 5 ] Mashimo, K. Homogeneous totally real submanifolds of $S^{6}$, Tsukuba J. Math., 9 (1985), 185-202.

[ 6 ] Sekigawa, K., Some CR-submanifolds in a 6-dimensioanl sphere, Tensor (N.S.), 6 (1984), 13-20.

[ 7 ] Mashimo, K., On the existence of 3-dimensional invariant submanifold of $S^{6}$, Topics in almost Hermitian geometry and related fields, Edited by Y. Matsushita et al., World Scientific, Singapore (2005), 186-189.

Present Addresses:

HideYa HaSHIMOTO

Department of Mathematics, Meijo University,

Shiogamaguchi, TenPaku, NAgOYa, 468-8502 JaPAN.

e-mail: hhashi@ccmfs.meijo-u.ac.jp

KaTSUYA MASHIMO

Department of Mathematics, Tokyo University of AgRiculture and TeChNology, KOGANEI, TOKYO, 184-8588 JAPAN.

e-mail: mashimo@cc.tuat.ac.jp 